\title{
MOLECULAR CONDUCTORS WITH TWO-CHAIN ORBITALS
}

\author{
H. Tajima, J. Shiraishi, and M. Kohmoto \\ Institute for Solid State Physics, University of Tokyo, Roppongi, Minato-ku, Tokyo 106, Japan
}

\begin{abstract}
We investigate a theoretical model applicable to molecular conductors, such as TTP and $\mathrm{M}(\mathrm{dmit})_{2}$ salts $[\mathrm{M}=\mathrm{Ni}, \mathrm{Pd}, \mathrm{Pt}]$, whose charge carriers orig inate from two kinds of molecular orbitals. The model Hamiltonian consists of two independent Hubbard chains. The degree of charge transfer between the two chains is calculated. The results qualitatively agree with some experimental data.
\end{abstract}

Keywords: A. organic crystals, D. electronic band structure 


\section{INTRODUCTION}

In most of the molecular conductors which exhibit metallic properties at low temperatures, charge carriers originate from molecular orbitals of one kind such as the highest occupied molecular orbital (HOMO) of a donor molecule or the lowest unoccupied molecular orbital (LUMO) of an acceptor molecule.[1] For example, the charge carriers of $\beta$-(BEDT$\mathrm{TTF})_{2} \mathrm{I}_{3}$ originate from the HOMO's of BEDT-TTF molecules while $\mathrm{I}_{3}^{-}$anions merely work as reservoirs of negative charges. In this type of molecular conductors, the chemical composition in principle determines the filling factor of the conduction band.

On the other hand, charge carriers in some molecular conductors originate from two kinds of molecular orbitals ("two-orbital" molecular conductors). Here we call one of the two kinds of molecular orbitals, having the larger orbital energy, as the upper orbital (UO), and the other, having the lower orbital energy, as the lower orbital (LO). The energy separation between the UO and LO is denoted by $\Delta$.

A molecular conductor composed of segregated columns of donor molecules and columns of acceptor molecules, such as TTF•TCNQ, may be considered as a two-component type of the "two-orbital" molecular conductor. The UO and LO in this type are the LUMO of an acceptor molecule and the HOMO of a donor molecule, respectively.

Another type of the "two-orbital" molecular conductor is a single-component one, where the UO and LO come from molecules of one kind. The salt of TTF $\left[\mathrm{Ni}(\mathrm{dmit})_{2}\right]_{2}$ is classified into this type. The UO and LO in this salt are the HOMO and LUMO of $\mathrm{Ni}(\mathrm{dmit})_{2}$, respectively.[2] For this type, one can estimate $\Delta$ approximately from the intra-molecular optical absorption for the molecule which possesses the UO and LO. The values of $\Delta$, thus determi ned for typical organic donors and acceptors, are listed in Table 1. In the "twoorbital" molecular conductors, the charge transfer between the two molecular orbitals is a controllable parameter, which one can expect to enrich their properties. In order to reveal them, we have studied a two-chain model.

\section{MODEL HAMILTONIAN}

The Hamiltonian which describes two-chained molecular orbitals interacting through the on-site Coulomb repulsion is written as

$$
\begin{aligned}
H & =H_{u}+H_{l}+T+Q, \\
H_{u} & \equiv \Delta \sum_{i, \sigma} a_{i \sigma}^{\dagger} a_{i \sigma}-t_{u u} \sum_{i, \sigma}\left(a_{i \sigma}^{\dagger} a_{i+1 \sigma}+\text { c.c. }\right)+U_{u u} \sum_{i} a_{i \uparrow}^{\dagger} a_{i \uparrow} a_{i \downarrow}^{\dagger} a_{i \downarrow}, \\
H_{l} & \equiv-t_{l l} \sum_{i, \sigma}\left(b_{i \sigma}^{\dagger} b_{i+1 \sigma}+\text { c.c. }\right)+U_{l l} \sum_{i} b_{i \uparrow}^{\dagger} b_{i \uparrow} b_{i \downarrow}^{\dagger} b_{i \downarrow}, \\
T & \equiv-t_{u l} \sum_{i, \sigma}\left(a_{i \sigma}^{\dagger} b_{i+1 \sigma}+\text { c.c. }\right)-t_{l u} \sum_{i, \sigma}\left(b_{i \sigma}^{\dagger} a_{i+1 \sigma}+\text { c.c. }\right), \\
Q & \equiv U_{u l} \sum_{i, \sigma, \sigma^{\prime}} a_{i \sigma}^{\dagger} a_{i \sigma} b_{i \sigma^{\prime}}^{\dagger} b_{i \sigma^{\prime}},
\end{aligned}
$$

where $a_{i \sigma}^{\dagger}$ and $a_{i \sigma}$ are the creation and annihilation operator of an electron at the $i$-th site with spin $\sigma$ in the UO; $b_{i \sigma}^{\dagger}$ and $b_{i \sigma}$ are those in the LO; $t_{u u}, t_{l l}, t_{u l}, t_{l u}$ are transfer integrals; 
and $U_{u u}, U_{l l}, U_{u l}$ are the on-site Coulomb repulsions. The model Hamiltonian (1) consists of two Hubbard chains $\left(H_{u u}, H_{l l}\right)$ and their couplings $(T, Q)$. If the couplings are neglected, the problem is reduced to the two Hubbard models which can be solved by Bethe ansatz. this approximation may be partially justified by the following considerations:

First, $T$ is anticipated to be unimportant until the charge transfer from the LO to the UO occurs, since $t_{u l}, t_{l u}$ are smaller than $t_{u u}, t_{l l}$, and negligible compared to $\Delta$.

Secondly,

$$
\begin{aligned}
Q= & \frac{U_{u l}}{2} \sum_{i}\left(a_{i \uparrow}^{\dagger} a_{i \uparrow}+a_{i \downarrow}^{\dagger} a_{i \downarrow}+b_{i \uparrow}^{\dagger} b_{i \uparrow}+b_{i \downarrow}^{\dagger} b_{i \downarrow}\right)^{2} \\
& -\frac{U_{u l}}{2} \sum_{i, \sigma}\left(a_{i \sigma}^{\dagger} a_{i \sigma}+b_{i \sigma}^{\dagger} b_{i \sigma}\right)-U_{u l} \sum_{i}\left(a_{i \uparrow}^{\dagger} a_{i \uparrow} a_{i \downarrow}^{\dagger} a_{i \downarrow}+b_{i \uparrow}^{\dagger} b_{i \uparrow} b_{i \downarrow}^{\dagger} b_{i \downarrow}\right),
\end{aligned}
$$

where the first term is proportional to the fluctuation of electron density at each site; the second term is proportional to the total electron number; and the third term is on-site Coulomb repulsion, which simply reduces the effective value of $U_{u u}$ in $H_{u}$ and that of $U_{l l}$ in $H_{l}$. Consequently, $Q$ can be neglected until the fluctuation of electron density becomes dominant.

Since $H_{u}$ and $H_{l}$ are now independent, the ground state is obtained by imposing the same chemical potential to the two chains. More explicitly one can obtain the ground state energy by minimizing $E \equiv E\left[(\mathrm{UO})^{N_{u}}\right]+E\left[(\mathrm{LO})^{N_{l}}\right]$ under the condition of $N_{u}+N_{l}=N_{t}$, where $E\left[(\mathrm{UO})^{N_{u}}\right]$ denotes the ground state energy for $H_{u}$ when $N_{u}$ electrons are in the UO, and $E\left[(\mathrm{LO})^{N_{l}}\right]$ denotes the corresponding energy for $H_{l}$ when $N_{l}$ electrons are in the LO. The total electron number in the system is $N_{t}$.

In the following calculation, we use the ground state energy per one site for the Hubbard chain. This energy, $\varepsilon_{H u b .}(u, n) \equiv E / N_{s}$, was originally derived by Lieb and Wu:[11,12,13]

$$
\begin{aligned}
\varepsilon_{H u b .}(u, n) & =-2 t \int_{-Q}^{Q} d k \cos k \rho(k), \\
\int_{-Q}^{Q} d k \rho(k) & =N / N_{s} \\
2 \pi \rho(k) & =1+\cos k \int_{-Q}^{Q} d k^{\prime} \rho\left(k^{\prime}\right) \frac{8 \pi}{u} R\left(\frac{4\left(\sin k-\sin k^{\prime}\right)}{u}\right), \\
R(x) & =\frac{1}{4 \pi} \int_{-\infty}^{\infty} \frac{\operatorname{sech}(\pi t / 2)}{1+(x+t)^{2}} d t
\end{aligned}
$$

where $N_{s}, N\left(<N_{s}\right)$ are the total number of sites and electrons; $n \equiv N / N_{s}$; and $u=U / t$, respectively. By solving these equations we can study the range $n<1$. For the range $n>1$, $\varepsilon_{H u b .}(u, n)$ is given by an identical equation,

$$
\varepsilon_{H u b .}(u, n)=\varepsilon_{H u b .}(u, 2-n)+U(n-1),
$$

coupled with Eq.(2.3). Since a closed form of (2.3) is not known, we have obtained following functions, $\varepsilon_{1}(u, n)$ and $\varepsilon_{2}(u, n)$ which approximate $\varepsilon_{H u b .}(u, n)$ :

$$
\begin{aligned}
\varepsilon_{H u b .}(u, n) & \cong \varepsilon_{1}(u, n) & & (0<n<1) \\
& \cong \varepsilon_{2}(u, n) & & (1<n<2)
\end{aligned}
$$


within the ansatz

$$
\begin{aligned}
& \varepsilon_{1}(u, n)=-A(u) t \sin \left(B(u) n+C(u) n^{2}+D(u) n^{3}\right) \\
& \varepsilon_{1}(u, n)=\varepsilon_{1}(u, 2-n)+U(n-1) ; \\
& A(u)=\frac{2}{\pi}\left(1+\left(1+P_{4} u+P_{5} u^{2}\right) \exp \left(-P_{6} u\right)\right) \quad(0<u<10) \\
& A(u)=\frac{2}{\pi}+P_{13} u^{-P_{14}} \quad(10<u<250) ; \\
& B(u)=2 / A(u) ; \\
& C(u)=P_{7} u /\left(1+P_{8} u^{2}+P_{9} u^{3}\right)+P_{10} u \quad(0<u<10) \\
& C(u)=P_{15} u^{-P_{16}}+P_{17} u^{-P_{18}} \quad(10<u<250) ; \\
& D(u)=\left(\pi / 2-B(u) n_{\max }-C(u) n_{\max }^{2}\right) / n_{\max }^{3} ;
\end{aligned}
$$

where

$$
\begin{gathered}
n_{\max }(u)=\frac{1}{2}\left(1+\left(1+P_{1} u+P_{2} u^{2}\right) \exp \left(-P_{3} u\right)\right) \quad(0<u<10), \\
n_{\max }(u)=\frac{1}{2}\left(1+P_{11} u^{-P_{12}}\right) \quad(10<u<250) ; \\
P_{1}=-0.08705 ; \quad P_{2}=0.01366 ; \quad P_{3}=0.23603 ; \\
P_{4}=-0.10512 ; \quad P_{5}=0.0207 ; \quad P_{6}=0.28767 ; \\
P_{7}=-0.14159 ; \quad P_{8}=0.06386 ; \quad P_{9}=0.00582 ; \\
P_{10}=-0.00903 ; \quad P_{11}=0.76063 ; \quad P_{12}=1.03065 ; \\
P_{13}=0.80627 ; \quad P_{14}=1.03467 ; \quad P_{15}=-1.3295 ; \\
P_{16}=0.75014 ; \quad P_{17}=0.12461 ; \quad P_{18}=0.39471
\end{gathered}
$$

The deviations of $\varepsilon_{1}(u, n)$ and $\varepsilon_{2}(u, n)$ from $\varepsilon_{H u b .}(u, n)$ are about $0.001 t$ in the range of $0<u<250$.

\section{RESULTS}

We take $U_{u u}=U_{l l}=U$ and $t_{u u}=t_{l l}=t$ for simplicity. Generalization to the other cases is straightforward.

a) $(\mathrm{UO})^{(1.5+x) N_{s}}(\mathrm{LO})^{(2-x) N_{s}}$

This is the case corresponding to the charge-transfer salts of $\mathrm{TTP}^{0.5+}$. [9] The parameter $x$ represents the degree of charge transfer from the LO to the UO. The ground-state energy per one site, $\varepsilon\left(\equiv E / N_{s}\right)$, is expressed by

$$
\begin{aligned}
\varepsilon & =\left(\varepsilon_{2}(U / t, 1.5+x)+(1.5+x) \Delta\right)+\varepsilon_{2}(U / t, 2-x) \\
& =1.5 U+(1.5+x) \Delta+\varepsilon_{1}(U / t, 0.5-x)+\varepsilon_{1}(U / t, x) .
\end{aligned}
$$

In what follows, $x$ is determined as the value which minimizes e for a given set of $U / t$ and $\Delta / t$ : 
i) If $\left.\frac{\partial \varepsilon}{\partial x}\right|_{x=+0}>0$, we have, $x=0$.

ii) Next, if $\left.\frac{\partial \varepsilon}{\partial x}\right|_{x=+0}<0$, we obtain $x$ as the solution of

$$
\frac{\partial \varepsilon}{\partial x}=\Delta-\left.\frac{\partial \varepsilon_{1}(U / t, n)}{\partial n}\right|_{n=0.5-x}+\left.\frac{\partial \varepsilon_{1}(U / t, n)}{\partial n}\right|_{n=x}=0 .
$$

Figure 1 shows the degree of charge transfer thus obtained. In the case of TTP salts, [9] the value of $\Delta / t$ is considered to be always larger than 2.[10,14] Therefore we conclude that the charge transfer from the LO to the UO does not occur in these salts. In fact, properties of these salts are consistent with the expectation given by the extended Hükel calculation where only the HOMO is taken into account.[14]

b) $(\mathrm{UO})^{(1+x) N s}(\mathrm{LO})^{(2-x) N_{s}}$

This is the case corresponding to the charge-transfer salts of $\mathrm{TTP}^{+}[9]$ and $\left[\mathrm{M}(\mathrm{dmit})_{2}\right]^{-}$ $(\mathrm{M}=\mathrm{Ni}, \mathrm{Pd}, \mathrm{Pt})$. The ground-state energy per site is expressed by

$$
\varepsilon=U+(1+x) \Delta+\varepsilon_{1}(U / t, 1-x)+\varepsilon_{1}(U / t, x) .
$$

The degree of charge transfer, $x$, is obtained as follows:

i) If $\left.\frac{\partial \varepsilon}{\partial x}\right|_{x=+0}>0$, we have $x=0$.

ii) Next if $\left.\frac{\partial \varepsilon}{\partial x}\right|_{x=+0}<0$, we obtain $x$ as the solution of

$$
\Delta-\left.\frac{\partial \varepsilon_{1}(U / t, n)}{\partial n}\right|_{n=1-x}+\left.\frac{\partial \varepsilon_{1}(U / t, n)}{\partial n}\right|_{n=x}=0 .
$$

Figure 2 shows the degree of charge transfer thus obtained. The ground state of the one-dimensional Hubbard model is insulating at half filling. Therefore, the two-chained Hubbard model (2.1) predicts an insulating state at $x=0$, metallic state for $x \neq 0$, and metal-insulator transition on the b oundary of these two regions.

In the case of $\mathrm{TTP}^{+}[9]$ salts, the value of $U / t$ is about 3.[10] Thus , from Fig 2a, one may expect that metallic state in these salts if $\Delta / t<3$. This condition is not unreasonable. In fact, Mori et al recently found the metallic behavior above $160 \mathrm{~K}$ in TTM-TTP• $\mathrm{I}_{3}$, which is a charge-transfer salt of $\mathrm{TTM}^{-\mathrm{TTP}^{+}}$. Although they did not consider the possibility of the charge transfer between the LO and UO[15], our calculation suggests that such charge transfer causes the metallic behavior of this salt above $160 \mathrm{~K}$.

c) $(\mathrm{UO})^{(0.5+x) N_{s}}(\mathrm{LO})^{(2-x) N_{s}}$

This is the case corresponding to the charge-transfer salts of $\left[\mathrm{M}(\mathrm{dmit})_{2}\right]^{0.5-}(\mathrm{M}=\mathrm{Ni}, \mathrm{Pd}$, $\mathrm{Pt})$. The ground-state energy per one site, $\varepsilon$, is expressed as follows:

$$
\begin{aligned}
& \varepsilon=U(1-x)+(0.5+x) \Delta+\varepsilon_{1}(U / t, 0.5+x)+\varepsilon_{1}(U / t, x) \quad(0<x<0.5) \\
& \varepsilon=0.5 U+(0.5+x) \Delta+\varepsilon_{1}(U / t, 1.5-x)+\varepsilon_{1}(U / t, x) \quad(0.5<x<0.75) .
\end{aligned}
$$

The degree of charge transfer, $x$, is determined as follows:

i) If $\left.\frac{\partial \varepsilon}{\partial x}\right|_{x=+0}>0$, we have $x=0$. 
ii) If $\left.\frac{\partial \varepsilon}{\partial x}\right|_{x=+0}<\left.0 \frac{\partial \varepsilon}{\partial x}\right|_{x=0.5-0}$, we obtain $x$ as the solution of

$$
-U+\Delta+\left.\frac{\partial \varepsilon_{1}(U / t, n)}{\partial n}\right|_{n=0.5+x}+\left.\frac{\partial \varepsilon_{1}(U / t, n)}{\partial n}\right|_{n=x}=0 .
$$

iii) If $\left.\frac{\partial \varepsilon}{\partial x}\right|_{x=0.5-0}<0<\left.\frac{\partial \varepsilon}{\partial x}\right|_{x=0.5+0}$, we have $x=0.5$.

iv) If $\left.\frac{\partial \varepsilon}{\partial x}\right|_{x=0.5+0}<0$, we obtain $x$ as the solution of

$$
\Delta-\left.\frac{\partial \varepsilon_{1}(U / t, n)}{\partial n}\right|_{n=1.5-x}+\left.\frac{\partial \varepsilon_{1}(U / t, n)}{\partial n}\right|_{n=x}=0 .
$$

Figure 3 shows the degree of charge transfer thus obtained. It should be noted that the state $x=0.5$ is stable in this model, as can be seen from Fig. 3a. This comes from the fact that $\partial \varepsilon_{H u b .}(u, n) / \partial n$ is discontinuous at $n=1$.

The present model Hamiltonian does not include any contribution from the electronphonon interaction. The interaction is expected to make the state, $x=0.5$, more stable by forming a gap in the middle of the half-filled UO band (Peierls transition). In this sense, the dimerized structure frequently found in $\mathrm{Pd}(\mathrm{dmit})_{2}$ and $\mathrm{Pt}(\mathrm{dmit})_{2}$ salts [16] may be considered as a result of Peierls transition.

d) $(\mathrm{UO})^{x N_{s}}(\mathrm{LO})^{(2-x) N_{s}}$

This is the case corresponding to the "two-orbital" molecular conductors of the twocomponent type, such as TTF $\bullet$ TCNQ. The ground-state energy per one site, $\varepsilon$, is expressed by

$$
\varepsilon=U(1-x)+x \Delta+2 \varepsilon_{1}(U / t, x) .
$$

The degree of charge transfer, $x$, is determined as follows:

i) If $\left.\frac{\partial \varepsilon}{\partial x}\right|_{x=+0}>0$, we have $x=0$.

ii) If $\left.\frac{\partial \varepsilon}{\partial x}\right|_{x=+0}<0<\left.\frac{\partial \varepsilon}{\partial x}\right|_{x=1-0}$, we obtain $x$ as the solution of

$$
-U+\Delta+\left.\frac{\partial \varepsilon_{1}(U / t, n)}{\partial n}\right|_{n=0.5+x}+\left.\frac{\partial \varepsilon_{1}(U / t, n)}{\partial n}\right|_{n=x}=0 .
$$

iii) If $\left.\frac{\partial \varepsilon}{\partial x}\right|_{x=1-0}<0$, we have $x=1$.

Figure 4 shows the degree of charge transfer thus obtained. This figure exhibits an insulating state at $x=0$ and $x=1$; metallic state for $0<x<1$. Although a "twoorbital" molecular conductor of a single-component type which exhibits metallic behavior has not been found, the present model predicts that synthesis of such compound is possible by choosing appropriate values of $U / t$ and $\Delta / t$.

\section{CONCLUSION}

We found that the on-site coulomb interaction plays quite an important role in the "twoorbital" molecular conductors. The effect of this interaction grows the electron density in 
the UO becomes smaller. The model gives a plausible explanation for the metallic behavior of TTM-TTP $\bullet \mathrm{I}_{3}$ and the strongly dimerized structure frequently observed in $\mathrm{Pd}(\mathrm{dmit})_{2}$ and $\mathrm{Pt}(\mathrm{dmit})_{2}$ salts. In spite of its simplicity, this model is versatile and applicable to many molecular conductors.

\section{Acknowledgment}

We deeply thank Prof. Kyuya Yakushi , Prof. Reizo Kato and Dr. David Lidsky for valuable discussions. This work was supported by the Grant-in-Aid for the Special Project Research on the "Novel Electronic State in Molecular Conductors" and by the Grant-in-Aid

for Scientific research (No. 08640733) from the Ministry of Education, Science and Culture, Japan. 


\section{REFERENCES}

[1] For example see, Ishiguro, T. and Yamaji, K., Organic Superconductors, SpringerVerlag, Berlin-Heidelberg, 1990.

[2] Canadell, E., Rachidi, I. E. I., Eavy, E., Pouget, J. P., Brossard, L., and Legros, J. P., J. Physique, 50, 1989, 2967.

[3] Yakushi, K., Iguchi, M., Katagiri, G., Kusaka, T., Ohta, T. and Kuroda, H., Bull. Chem. Soc. Jpn., 54, 1981, 348.

[4] Naito, T. and Tajima, H., unpublished results.

[5] Sugano, T., Yakushi, K. and Kuroda, H., Bull. Chem. Soc. Jpn., 51, 1978, 1041.

[6] Yakushi, K., Tajima, H., Ida, T., Tamura, M., Hayashi, H., Kuroda, H., Kobayashi, A., Kobayashi, H. and Kato, R., Synthetic Metals, 24, 1988, 301.

[7] Yoshitake, M., Yakushi, K., Kuroda, H., Kobayashi, A., Kato, R. and Kobayashi, H., Bull. Chem. Soc. Jpn., 61, 1988, 1115.

[8] Tajima, H., Tamura, M., Kuroda, H., Mori, T. and Inokuchi, H., Bull. Chem. Soc. Jpn., 63, 1990, 538.

[9] In this paper we use "TTP" as a generic name of BDT-TTP and its derivatives, such as TTM-TTP, TMET-TTP.

[10] Tajima, H., Arifuku, M., Ohta, T., Mori, T., Misaki, Y., Yamabe, T., Mori, H. and Tanaka, S., Synthetic Metals, 71, 1995, 1951.

[11] Shiba, H., Phys Rev., B6, 1972, 930.

[12] Lieb, E. H. and Wu, F. Y., Phys. Rev. Lett, 20, 1968, 1445.

[13] Takahashi, M., Progr. Theoret. Phys. (Kyoto), 42, 1969, 1098; 43, 1970, 1619.

[14] See Mori, T., Kawamoto, T., Misaki, Y., Kawakami, K., Fujiwara, H., Yamabe, T., Mori, H. and Tanaka, S., Mol. Cryst. Liq. Cryst., 284, 1996, 271; and references therein.

[15] Mori, T., Inokuchi, H., Misaki, Y., Yamabe, T., Mori, H. and Tanaka, S., Bull. Chem. Soc. Jpn., 67, 1994, 661.

[16] For example see the following review: Cassoux, P., Valade, L., Kobayashi, H., Kobayashi, A., Clark, R. A. and Underhill, A. E., Coord. Chem. Rev., 110, 1991, 115. 


\section{TABLES}

s.:determined from an absorption spectrum of solution

c.:determined from an absorption spectrum of a single crystal

\begin{tabular}{lllll}
\hline \hline & $\Delta(\mathrm{eV})$ & UO & LO & Ref. \\
$\begin{array}{l}\text { acceptor } \\
\text { TCNQ }^{-}\end{array}$ & 1.3 & LUMO & HOMO & {$[3]^{s .}$} \\
Ni(dmit $)_{2}^{-}$ & 1.1 & LUMO & HOMO & {$[4]^{s .}$} \\
Pd(dmit $)_{2}^{-}$ & 0.9 & LUMO & HOMO & {$[4]^{s .}$} \\
& & & & \\
donor & 2 & HOMO & 2nd HOMO & {$[5]^{s .}$} \\
TTF $^{+}$ & 1.7 & HOMO & 2nd HOMO & {$[6]^{s .}$} \\
BPDT-TTF $^{+}$ & 1.4 & HOMO & 2nd HOMO & {$[7]^{c .}$} \\
BMDT-TTF $^{+}$ & 1.4 & HOMO & 2nd HOMO & {$[8]^{c .}$} \\
BEDT-TTF $^{+}$ & 0.9 & HOMO & 2nd HOMO & {$[10]^{c .}$} \\
TTP $^{+}[9]$ & \hline
\end{tabular}

TABLE I. The energy separation between the UO and LO. The molecular orbitals (LUMO, HOMO, and 2nd HOMO) are defined in the neutral state of an isolated molecule. 


\section{FIGURES}

Fig. 1 (a)The degree of charge transfer $(x)$ obtained for the two-chained orbitals, $(\mathrm{UO})^{(1.5+x) N_{s}}(\mathrm{LO})^{(2-x) N_{s}}$. (b)The regions for $x=0$ and for $0<x<0.25$.

Fig. 2 (a) The degree of charge transfer $(x)$ obtained for the two-chained orbitals, $(\mathrm{UO})^{(1+x) N_{s}}(\mathrm{LO})^{(2-x) N_{s}}$. (b)The regions for $x=0$ and for $0<x<0.5$.

Fig. 3 (a)The degree of charge transfer $(x)$ obtained for the two-chained orbitals, $(\mathrm{UO})^{(0.5+x) N_{s}}(\mathrm{LO})^{(2-x) N_{s}}$. (b)The regions for $x=0 ; 0<x<0.5 ; x=0.5$; and $0.5<x<0.75$.

Fig. 4 (a)The degree of charge transfer $(x)$ obtained for the two-chained orbitals, $(\mathrm{UO})^{x N_{s}}(\mathrm{LO})^{(2-x) N_{s}}$. (b)The regions for $x=0 ; 0<x<1$; and $x=1$. 


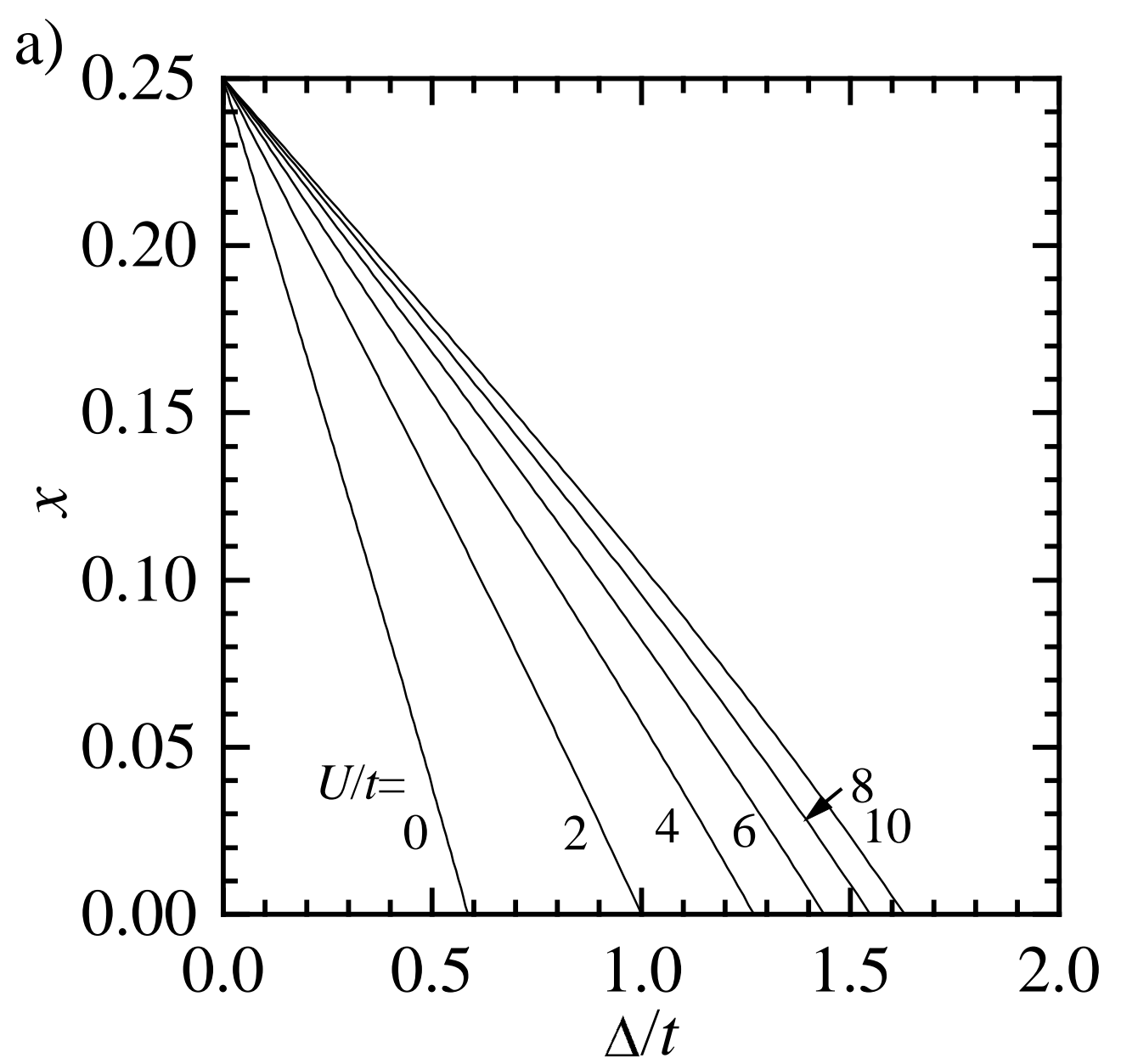

$1 \mathrm{D}$ chain $\quad(\mathrm{UO})^{(1.5+x) N_{s}}(\mathrm{LO})^{(2-x) N_{s}}$

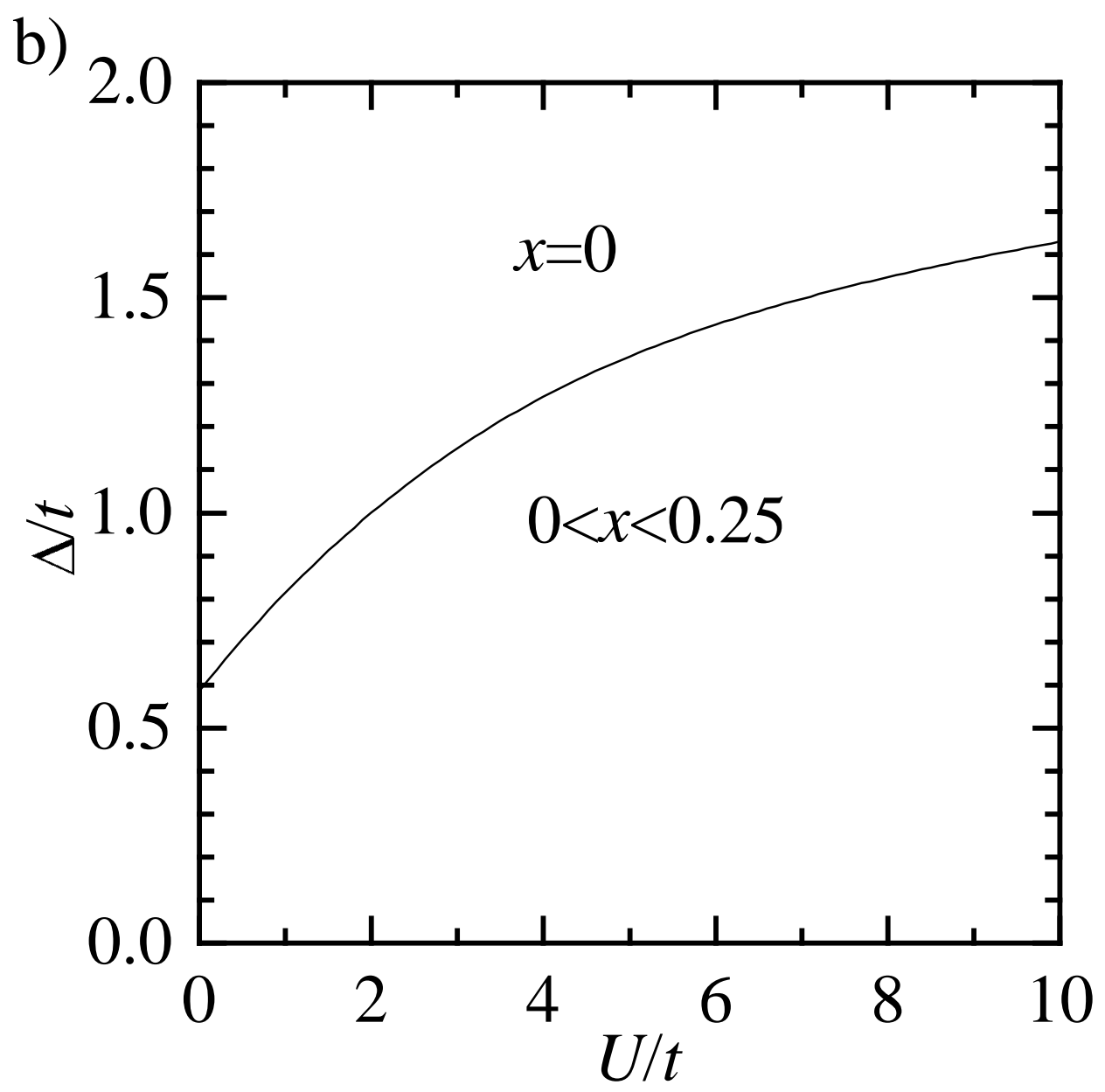

Fig. 1 Tajima et al 
a)

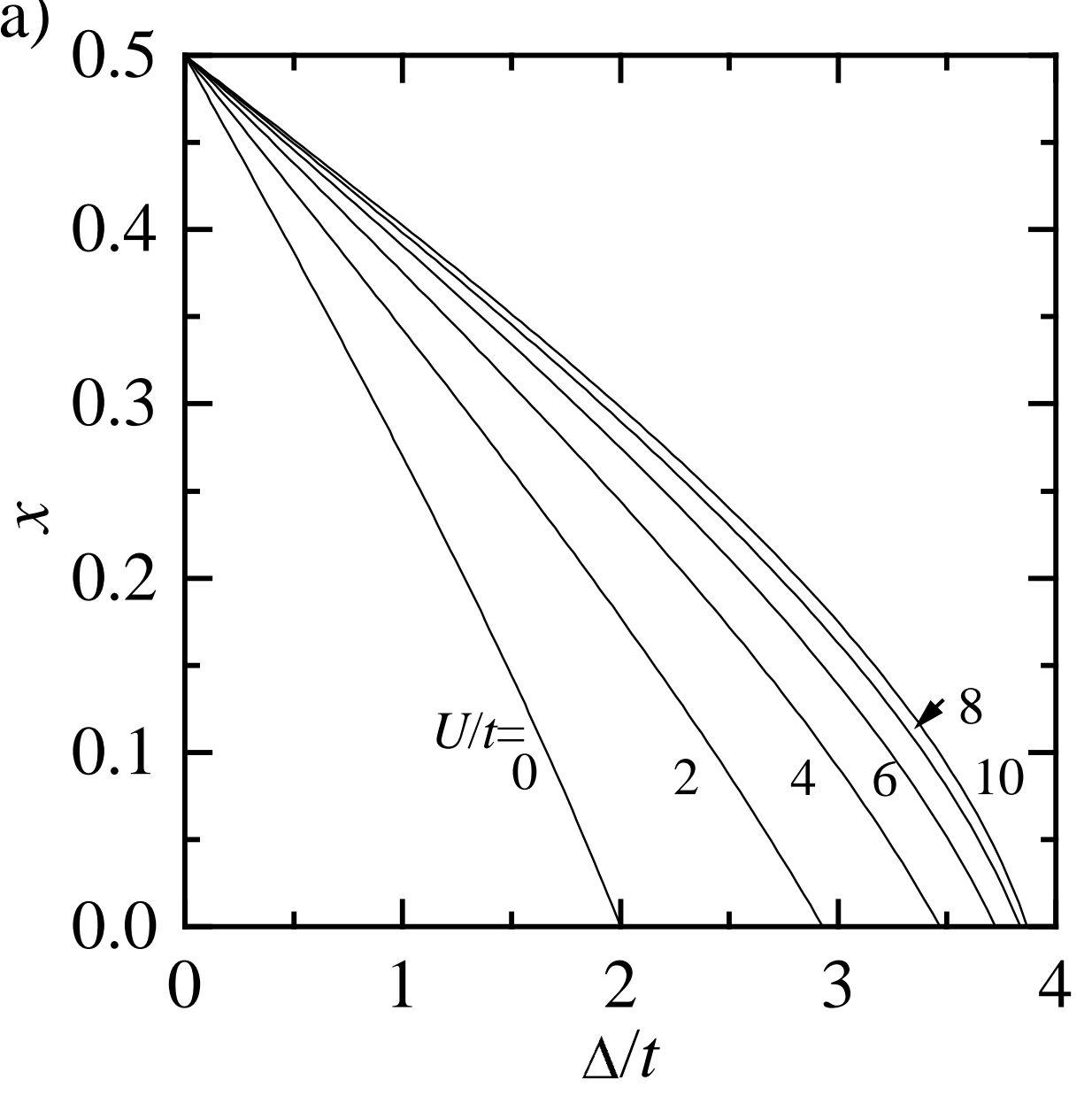

1D chain b) 4

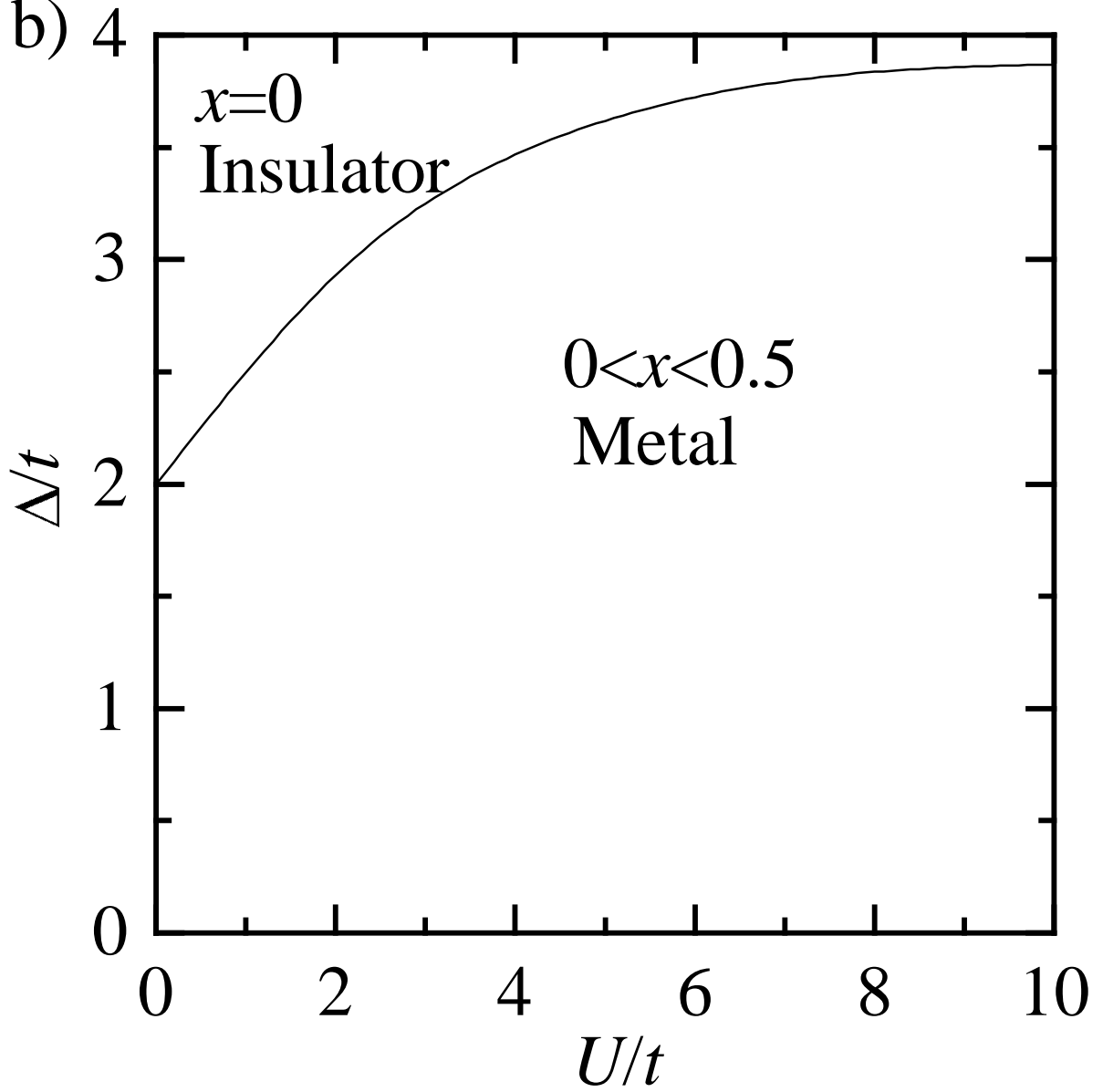

$(\mathrm{UO})^{(1+x) N_{s}(\mathrm{LO})^{(2-x) N_{s}}}$

Fig. 2 Tajima et al 

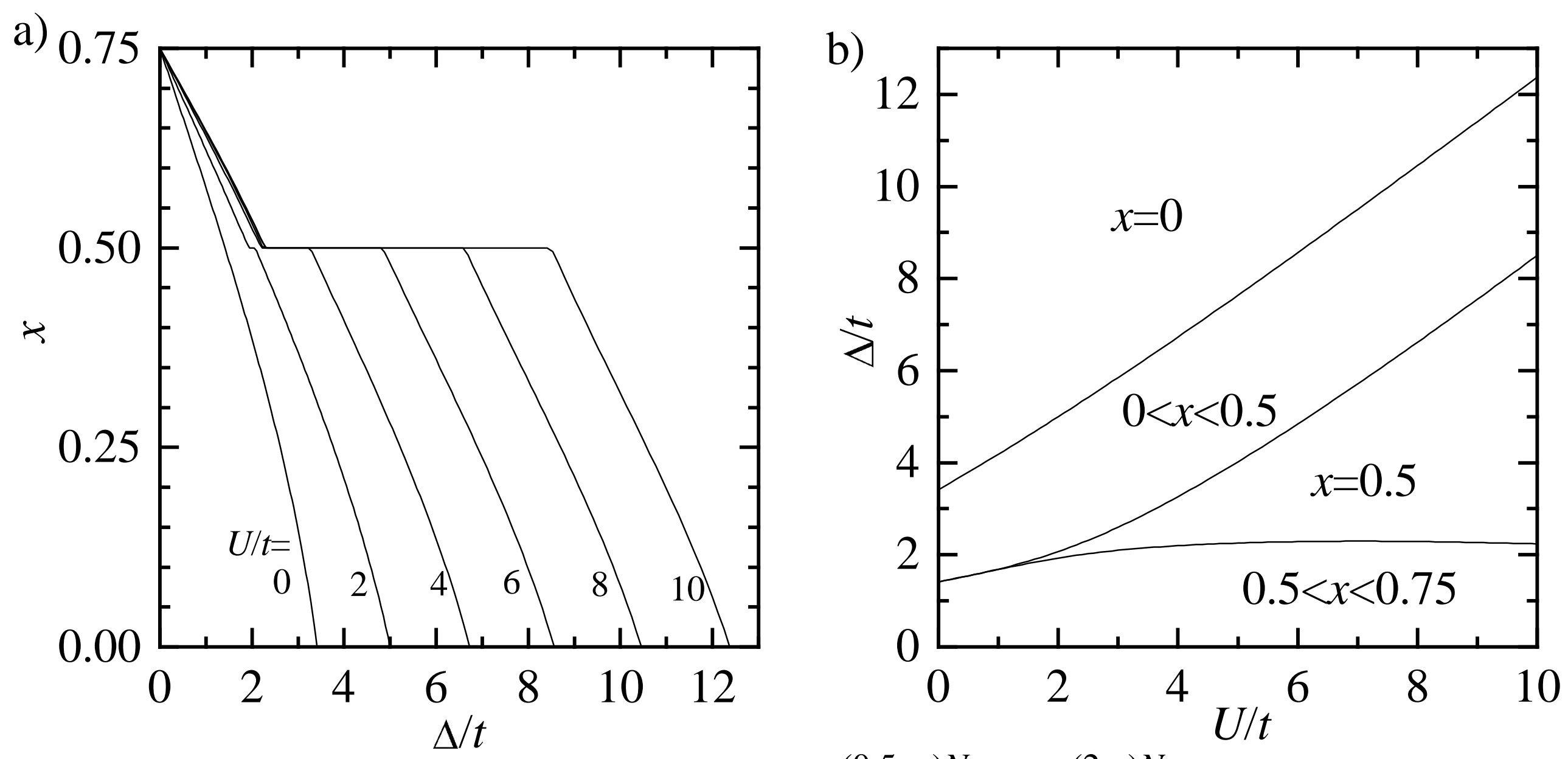

$1 \mathrm{D}$ chain $\quad(\mathrm{UO})^{(0.5+x) N_{s}(\mathrm{LO})^{(2-x) N_{s}}}$

Fig. 3 Tajima et al 

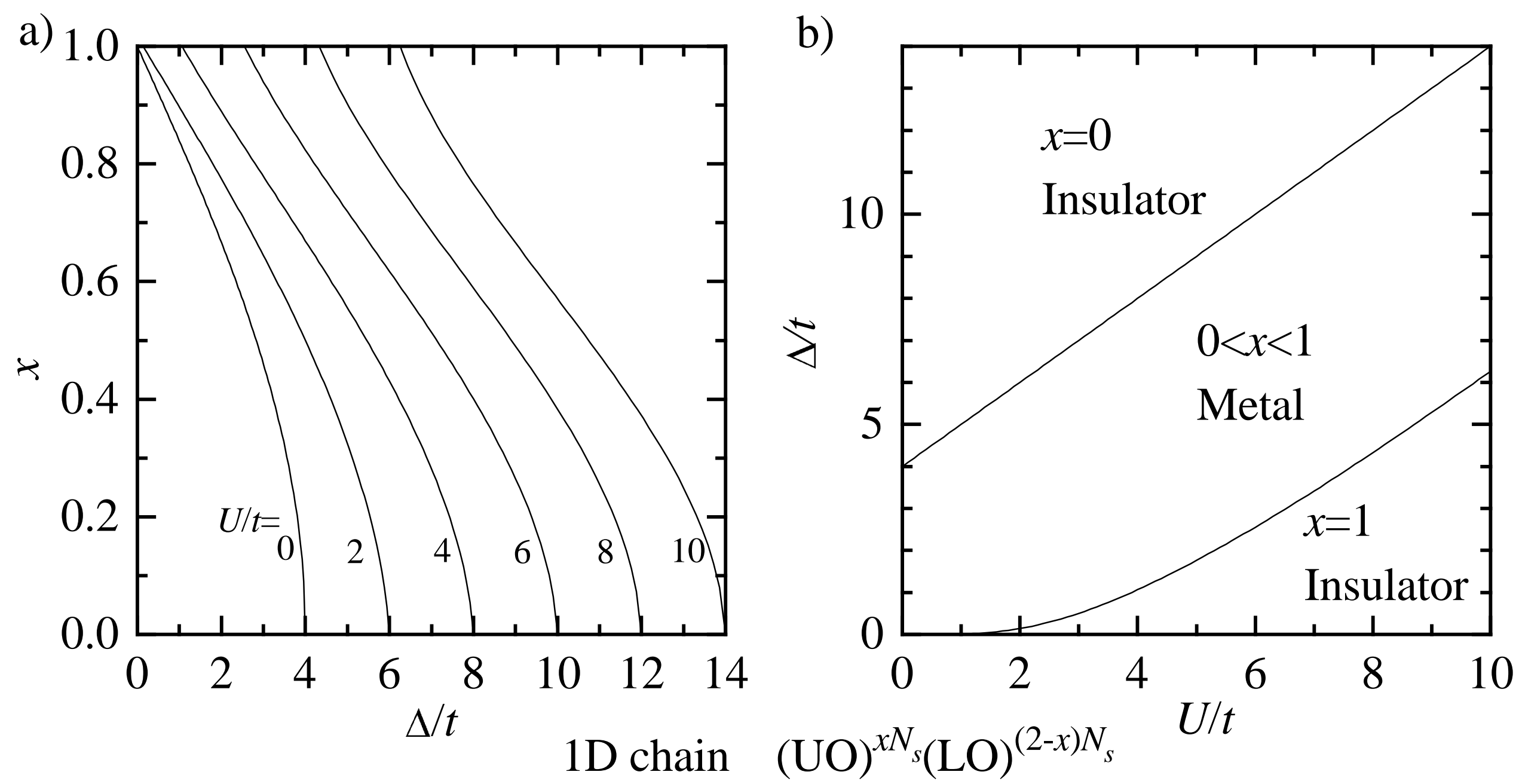

Fig. 4 Tajima et al 